\title{
Integrated Antenna-Battery for Low-profile Short Range Communications
}

\author{
Michael A. Woods ${ }^{1,2}$, Osman O. Rakibet ${ }^{1}$, Paul R. Young ${ }^{1}$, Robert Luck ${ }^{2}$, Maria L. Alfredsson ${ }^{2}$ and John C. \\ Batchelor $^{1}$ \\ ${ }^{1}$ School of Engineering: University of Kent, Canterbury, Kent, UK, maw44@kent.ac.uk \\ ${ }^{2}$ School of Physical Sciences: University of Kent, Canterbury, Kent, UK
}

\begin{abstract}
An integrated antenna-battery was designed in order to function at $2.45 \mathrm{GHz}$, with short range Bluetooth applications considered. The model was derived from a previous study in which an integrated system was first explored. The tan $\delta$ was investigated for the substrate and this new structure was found to give favourable gains and efficiencies compared to the previous study. Experimental parameters were also applied to the model in the presence of a dielectric material. These experimental parameters gave improved results over the initial assumptions.
\end{abstract}

Index Terms - antenna, low-profile, battery, short range.

\section{INTRODUCTION}

With the advent of new proximity location systems operating with Bluetooth links to smart phones, for instance the 'Tile' tag [1], there is interest in creating extreme lowprofile and integrated antenna/battery components for use at $2.45 \mathrm{GHz}$. The battery and antenna are typically the bulkiest components in a transceiver system and it is therefore of interest to combine them and ideally use novel digital fabrication technologies to realize them using low profile conducting polymer films.

A standard lithium ion battery is comprised of two electrodes, an anode and cathode. Lithium ions will be transported between the two via an electrolyte solution [2]. Metallic current collectors present either side of the two electrodes allow for connection to an external circuit.

A requirement for the new low-profile integrated battery is that it be non-metallic. This means that the battery cannot have the metallic hermetic seal that is standard, and thus the metallic current collectors must be replaced with another highly conductive material, with conducting polymers being the main consideration. Conducting polymers are already in use in batteries and have shown promising results thus far [3]. One polymer of keen interest in electronics is PEDOT:PSS, a copolymer which has demonstrated conductivities of over $3000 \mathrm{~S} / \mathrm{cm}$ in some cases upon treatment with sulphuric acid [4]. With conductivity values just a few orders below those of bulk metals such as copper, polymer electrolytes such as PEDOT:PSS are a viable alternative as current collectors in a non-metallic battery.

\section{INTEGRATED BATTERY-ANTENNA STRUCTURE}

Our antenna design is a symmetric slot antenna cut into a conducting film, with an electrolyte substrate underneath, followed by a second conducting layer, also with slots present. The conducting films are used to represent the anode and cathode of a solid state battery. Furthermore, they will also act as the battery terminals, providing the electrical conductivity needed for the battery to function.

Fig. 1 shows the integrated battery/antenna design proposed.
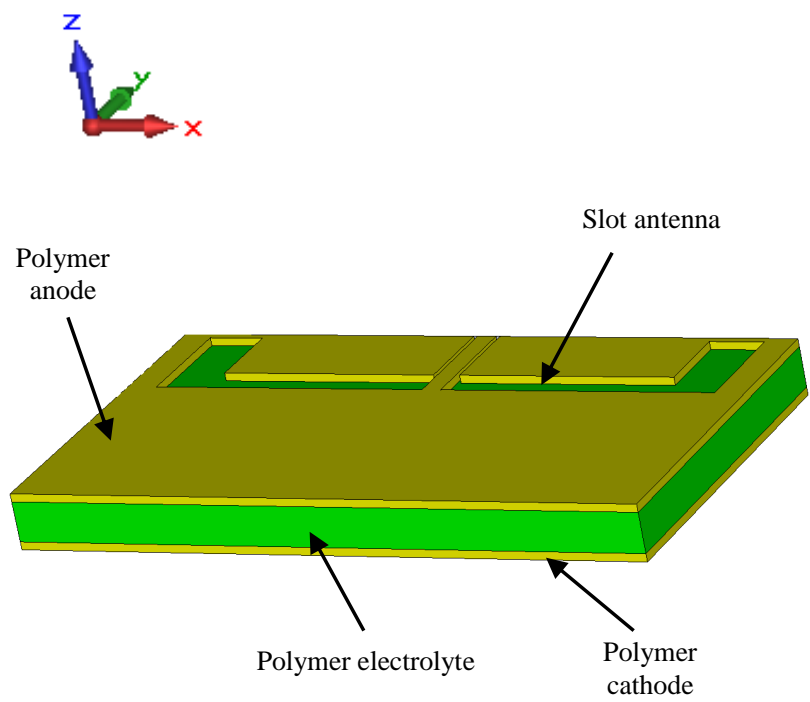

Fig. 1: Integrated battery/antenna design for Bluetooth application.

The CST (Computer Simulation Technology) Microwave Studio package transient solver was used to perform the required simulations for this investigation. The slot dipole antenna consisted of two symmetric slots cut into an upper conducting plane. The antenna structure is derived from that of [5], and is based on Nithisopa's CPW-fed slot dipole [6], and redesigned for the $2.45 \mathrm{GHz}$ band. The substrate was of a thickness of $0.3 \mathrm{~mm}$, with a relative permittivity $\left(\varepsilon_{\mathrm{r}}\right)$ of 3 . 


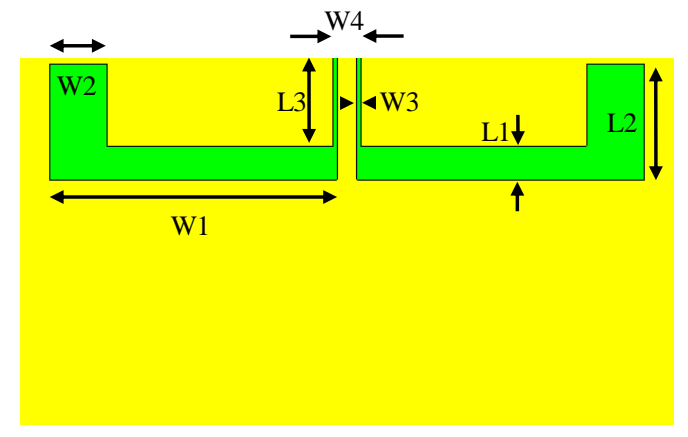

(a)

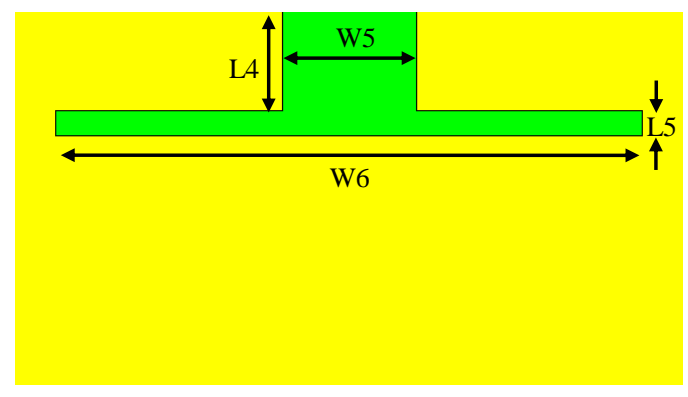

(b)

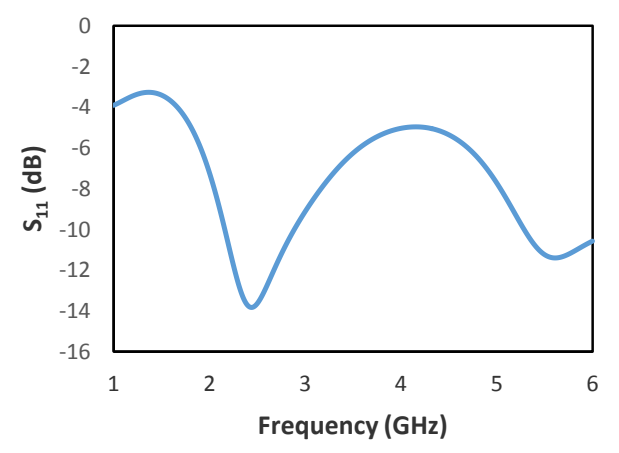

(c)

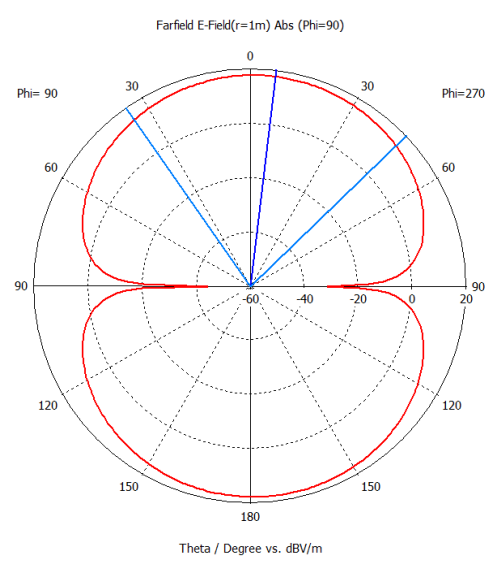

(d)

Fig. 2: (a) Dimensions for slot antenna, top plane (b) and bottom plane, (c) Simulated $\mathrm{S}_{11}$ and (d) Simulated far-field at $2.45 \mathrm{GHz}$.
Both the upper and lower ground planes had a thickness of $0.018 \mathrm{~mm}$. The dipole slots in this particular structure were symmetrical, with a width and length (W1) and (L1), of $35 \mathrm{~mm}$ and $4.1 \mathrm{~mm}$ respectively. The end caps, or folded sections, on each slot were each of length (L2) and width (W2), $14.1 \mathrm{~mm}$ and $7 \mathrm{~mm}$. The width of the feed slots (W3) was $0.5 \mathrm{~mm}$, with the gap between the two (W4) being $2.4 \mathrm{~mm}$. The CPW feed line for the antenna consisted of a length (L3) of $11 \mathrm{~mm}$. Slots were also present on the bottom ground plane with the slot beneath the co-planar wave guide feed of a length (L4) of $12 \mathrm{~mm}$ and width (W5) of $16 \mathrm{~mm}$. The slot beneath the dipole was of a length (L5) $3 \mathrm{~mm}$ and width (W6) $70 \mathrm{~mm}$.

The antenna itself had an overall width of $80 \mathrm{~mm}$ with an overall length of $45 \mathrm{~mm}$. The substrate was initially simulated with a low-loss $\tan \delta$ value of 0.0009 . A conductivity value of $1000 \mathrm{~S} / \mathrm{m}$ was applied to the two conducting planes as might be expected for a good conducting polymer electrolyte. Figure 2 (a) and (b) show the upper and lower plane views of the structure along with the associated length and width parameters, followed by (c), the simulated $S_{11}$ results, and (d) the associated far-field results at $2.45 \mathrm{GHz}$.

From the $S_{11}$ results, we can see that the structure is noticeably lossy with an out of band loss of about $4 \mathrm{~dB}$. However, with our low range application in mind, this is not a concern. The $-10 \mathrm{~dB}$ fractional bandwidth was calculated to be about $28 \%$ and the antenna simulated total efficiency was $-2.3 \mathrm{~dB}$ with a gain of $2.8 \mathrm{~dB}$. This can be compared to that of [5] where efficiencies of about $-2.2 \mathrm{~dB}$ and gains of about $3.3 \mathrm{~dB}$ at $2.6 \mathrm{GHz}$ were obtained.

The loss tangent of the substrate was then altered from 0.0009 to 0.9 , as might be anticipated for a battery electrolyte. Here, we observed a reduction in gain to $0.3 \mathrm{~dB}$ and a reduction in efficiency to $-4.8 \mathrm{~dB}$. Once again, comparing this to [5] where an efficiency of $-7.3 \mathrm{~dB}$ at $2.6 \mathrm{GHz}$ was simulated for a loss tangent of 0.9 , a noticeable improvement was achieved. Table I shows a summary of the results obtained from the initial structure of Fig. 2 compared with the results obtained from the previous study [5].

This low profile slotted design is affected by dielectric loading when mounted on structures and a simulated investigation was carried out to ascertain how the antenna match and efficiency were altered when the antenna-battery was placed on a dielectric block of relative permittivity 2.3 and $\tan \delta 0.09$.

The antenna bandwidth and efficiency underwent a change upon dielectric loading and a few adjustments were made to the antenna in order to allow it to function again at $2.45 \mathrm{GHz}$. The width of the antenna was reduced from $80 \mathrm{~mm}$ to $75 \mathrm{~mm}$, while the width of the slots (W6) beneath the dipole were reduced from $70 \mathrm{~mm}$ to $55 \mathrm{~mm}$. The substrate material parameters were kept constant with the $\tan \delta$ at 0.9 . When mounted onto the dielectric with the altered dimensions, a bandwidth of $55 \%$ was observed, with an efficiency of $-6.3 \mathrm{~dB}$ and a gain of $-1.7 \mathrm{~dB}$. This still gives a slight improvement in gain and efficiencies compared to the results of [5] in which the structure was not mounted onto any dielectric material. 
TABLE I

EFFECT OF LOSS TANGENT OF SUBSTRATE UPON BANDWIDTH, GAIN AND EFFICIENCY

\begin{tabular}{cccccc}
\hline \hline Tan $\delta$ & $\begin{array}{c}\text { Fractional } \\
\text { Bandwidth } \\
(\%)\end{array}$ & $\begin{array}{c}\text { Gain } \\
(\mathrm{dB}) \\
(\text { Fig.2) }\end{array}$ & $\begin{array}{c}\text { Efficiency } \\
(\mathrm{dB})\end{array}$ & $\begin{array}{c}\text { Gain } \\
(\mathrm{dB})\end{array}$ & $\begin{array}{c}\text { Efficiency } \\
(\mathrm{dB})[5]\end{array}$ \\
\hline 0.0009 & 28 & 2.8 & -2.3 & 3.3 & -2.2 \\
0.9 & 44 & 0.3 & -4.8 & -1.8 & -7.3 \\
\hline \hline
\end{tabular}

\section{SAMPLE MEASUREMENT}

It was desirable to create a sample of material as would be used in the polymer battery to form an electrode. A pellet of PEDOT:PSS, a highly conductive polymer electrolyte, was synthesized for this purpose. Aliquots $(\sim 30 \mathrm{ml})$ of conductive grade PEDOT-PSS (3\% weight dispersion in water) were pipetted into five separate sample dishes. The samples were dried for 48 hours at $55^{\circ} \mathrm{C}$ under atmospheric pressure. The precipitate was collected from each sample and processed within a ball-mill (800rpm, 40mins). A fine powdered sample was produced and subsequently pressed into a pellet by use of a $25 \mathrm{~mm}$ pellet-press. The dimensions of the pellet were a width of $25 \mathrm{~mm}$ and a thickness of $3 \mathrm{~mm}$.

The permittivity of the PEDOT:PSS sample was measured by placing a Coplanar Waveguide (CPW) track on top of the material. This was achieved by etching a $24 \mathrm{~mm}$ long CPW line onto a Mylar (polyester) sheet which was then placed directly on top of the sample. The gap between the signal and ground lines was set to $1.5 \mathrm{~mm}$ to ensure good penetration of the field into the material at the frequency range of measurement. An Anritsu 37397C network analyser with onwafer calibration was used to measure the S-parameters up to $20 \mathrm{GHz}$. The line proved to be well matched to $50 \Omega$ allowing the phase constant and effective permittivity of the line to be calculated directly from the $S_{21}$ measurement. Using the approximation that the effective permittivity of a CPW is the average of the air and dielectric region allowed a figure of $\varepsilon_{\mathrm{r}}=$ 4.3 and $\tan \delta=0.13$ for the relative permittivity and loss tangent to be obtained.

These measured material values were applied to the battery/antenna substrate and simulated. The underlying dielectric material was still present and remained unchanged. The permittivity of the antenna substrate was increased from 3 to 4.3 , while the $\tan \delta$ value of 0.9 was reduced to 0.13 . The main observations were a slight reduction in bandwidth, which is to be expected when decreasing the loss tangent. More importantly, an improvement in efficiency with a value of $-4.63 \mathrm{~dB}$ was observed, with an increase in gain to $0.02 \mathrm{~dB}$. Table II gives a summary of the comparison of simulation results for the antenna mounted onto the dielectric block with both the initial assumptions and the results obtained from the experimental data.

TABLE II

COMPARISON OF INITIAL ASSUMPTIONS FOR ANTENNA WITH PARAMETERS OBTAINED FROM EXPERIMENT IN THE PRESENCE OF DIELECTRIC MATERIAL

\begin{tabular}{ccccc}
\hline \hline $\begin{array}{c}\text { Permittivity } \\
\left(\varepsilon_{\mathrm{r}}\right)\end{array}$ & Tan $\delta$ & $\begin{array}{c}\text { Bandwidth } \\
(\%)\end{array}$ & Gain $(\mathrm{dB})$ & $\begin{array}{c}\text { Efficiency } \\
(\mathrm{dB})\end{array}$ \\
\hline 3 & 0.9 & 55 & -1.7 & -6.3 \\
4.3 & 0.13 & 44 & 0.02 & -4.6 \\
\hline \hline
\end{tabular}

\section{CONCLUSION}

The feasibility of producing extremely low profile composite polymer-battery antenna devices for active short range systems such as blue tooth has been indicated by simulation. Material measurements of a candidate polymer sample indicate that losses may not be prohibitively high, though further studies using appropriately treated polymer samples will be required before validation with antenna structures. This technology is proposed for use in short range location finding and security applications where very low profile or covert tags may be beneficial.

\section{ACKNOWLEDGMENT}

We thank ESPRC for providing Michael Woods' $\mathrm{PhD}$ studentship.

\section{REFERENCES}

[1] http://www.thetileapp.com/

[2] J.M. Tarascon and M. Armand, Issues and challenges facing rechargeable lithium batteries, Nature, Vol. 414, pp 359-367, 2001

[3] Takao Nagatomo, Chiaki Ichikawa, and Osamu Omoto. All-plastic batteries with polyacetylene electrodes, Journal of the Electrochemical Society, Vol. 134, pp 305-308, 1987

[4] Yijie Xia, Kuan Sun, and Jianyong Ouyang, Solution-processed metallic conducting polymer films as transparent electrode of optoelectronic devices, Adv. Mater., Vol. 24, pp 2436-2440, 2012

[5] M. Woods and J. Batchelor, "Low-profile Slot Antenna integrated with a thin polymer non-metallic battery", IEEE Loughborough Antenna \& Propagation Conference 2013

[6] K. Nithisopa, J. Nakasuwan, N. Songthanapitak, N. Anantrasirichai, and T. Wakabayashi, Design CPW fed slot antenna for wideband applications, Piers Online, Vol. 3, No. 7, pp 1124-1127, 2007 